\title{
Identification of the Multiresistance Gene poxt $A$ in Oxazolidinone-Susceptible Staphylococcus haemolyticus and Staphylococcus saprophyticus of Pig and Feed Origins
}

\author{
Lin Chen 1,2,3, + , Jian-Xin Hu ${ }^{1,2,+}$, Chang Liu ${ }^{1,2}$, Jiao Liu ${ }^{1,2}$, Zhen-Bao Ma 1,2, Zi-Yun Tang 1,2, Ya-Fei Li ${ }^{3, *}$ \\ and Zhen-Ling Zeng ${ }^{1,2, *}$ \\ 1 College of Veterinary Medicine, Guangdong Provincial Key Laboratory of Veterinary Pharmaceutics \\ Development and Safety Evaluation, National Risk Assessment Laboratory for Antimicrobial Resistance of \\ Animal Original Bacteria, South China Agricultural University, Guangzhou 510642, China; \\ chenl@stu.scau.edu.cn (L.C.); 20192027012@stu.scau.edu.cn (J.-X.H.); 20182027011@stu.scau.edu.cn (C.L.); \\ 20182027012@stu.scau.edu.cn (J.L.); mazhenbao@stu.scau.edu.cn (Z.-B.M.); \\ 20182027026@stu.scau.edu.cn (Z.-Y.T.) \\ 2 Guangdong Laboratory for Lingnan Modern Agriculture, South China Agricultural University, \\ Guangzhou 510642, China \\ 3 Public Monitoring Center of Agro-Product of Guangdong Academy of Sciences, Guangzhou 510640, China \\ * Correspondence: liyafei@gdaas.cn (Y.-F.L.); zlzeng@scau.edu.cn (Z.-L.Z.); Tel./Fax: +86-20-85284896 (Z.-L.Z.) \\ + These authors contributed equally to this article.
}

check for updates

Citation: Chen, L.; Hu, J.-X.; Liu, C.; Liu, J.; Ma, Z.-B.; Tang, Z.-Y.; Li, Y.-F.; Zeng, Z.-L. Identification of the Multiresistance Gene poxt $A$ in Oxazolidinone-Susceptible Staphylococcus haemolyticus and Staphylococcus saprophyticus of Pig and Feed Origins. Pathogens 2021, 10, 601. https://doi.org/10.3390/ pathogens10050601

Academic Editor: Jilei Zhang

Received: 6 March 2021

Accepted: 10 May 2021

Published: 14 May 2021

Publisher's Note: MDPI stays neutral with regard to jurisdictional claims in published maps and institutional affiliations.

Copyright: (c) 2021 by the authors. Licensee MDPI, Basel, Switzerland. This article is an open access article distributed under the terms and conditions of the Creative Commons Attribution (CC BY) license (https:/ / creativecommons.org/licenses/by/ $4.0 /)$.

\begin{abstract}
Previous studies on the prevalence and transmission mechanism of oxazolidinone resistance gene poxt $A$ in CoNS are lacking, which this study addresses. By screening 763 CoNS isolates from different sources of several livestock farms in Guangdong, China, 2018-2020, we identified that the poxt $A$ was present in seven CoNS isolates of pig and feed origins. Species identification and multilocus sequence typing (MLST) confirmed that seven poxtA-positive CoNS isolates were composed of five ST64-Staphylococcus haemolyticus and two Staphylococcus saprophyticus isolates. All poxtA-positive Staphylococcus haemolyticus isolates shared similar pulsed-field gel electrophoresis (PFGE) patterns. Transformation assays demonstrated all poxt $A$-positive isolates were able to transfer poxt $A$ gene to Staphylococcus aureus RN4220. S1-PFGE and whole-genome sequencing (WGS) revealed the presence of poxtA-carrying plasmids in size around $54.7 \mathrm{~kb}$. The plasmid pY80 was 55,758 bp in size and harbored the heavy metal resistance gene $c z c D$ and antimicrobial resistance genes, poxt $A, \operatorname{aad} D, \operatorname{fex} B$ and tet(L). The regions (IS1216E-poxtA-IS1216E) in plasmid pY80 were identified in Staphylococcus spp. and Enterococcus spp. with different genetic and source backgrounds. In conclusion, this was the first report about the poxtA gene in Staphylococcus haemolyticus and Staphylococcus saprophyticus, and IS1216 may play an important role in the dissemination of poxt $A$ among different Gram-positive bacteria.
\end{abstract}

Keywords: poxtA; CoNS; transformation; plasmids; antimicrobial; heavy metal; IS1216

\section{Introduction}

Coagulase negative staphylococci (CoNS) are one of the most common opportunistic pathogens found on human skin and mucous membranes as a component of normal flora [1,2]. Besides their role in keeping homeostasis, CoNS have been involved in a series of infectious processes, ranging from nosocomial infections to livestock bacterial sepsis and mastitis $[3,4]$. In addition to their virulence, the emergence of antibiotic resistance in CoNS and then horizontal dissemination among staphylococci should be alarming. The increasing drug resistance of CoNS significantly limited the treatment options [5,6]. Among CoNS, Staphylococcus haemolyticus is the second most frequently isolated from human blood culture and Staphylococcus saprophyticus is one of the most common pathogens responsible for community urinary tract infections $[5,7]$. 
Oxazolidinones such as linezolid and tedizolid are antibacterially active against Grampositive pathogens including methicillin-resistant Staphylococcus aureus (MRSA), methicillinresistant CoNS and vancomycin-resistant enterococci (VRE) [8]. However, the discovery of transferable oxazolidinone resistance genes such as $c f r, c f r(B), c f r(C), \operatorname{optr} A$ and $\operatorname{poxt} A$ as well as the mutations in 23S rRNA and ribosomal proteins L3 and L4 challenged the clinical use of oxazolidinones [9]. Worriedly, linezolid-resistant staphylococci have been detected worldwide [10]. The plasmid-mediated oxazolidinone resistance genes including $c f r$ and optrA spread among a number of bacterial species of different origins around the world shortly after they were reported [11]. The fact that $c f r$ and $\operatorname{optr} A$ genes can be selected by phenicols and other ribosomal-targeted drugs that are widely used in livestock and veterinary hospitals is closely associated with global spread of the resistance genes [12-14]. The recently described plasmid-mediated oxazolidinone resistance gene poxt $A$ could decrease susceptibility to phenicols and tetracyclines, so the poxt $A$ gene posed a threat to disseminate in bacteria from animal setting $[15,16]$. The poxt $A$ gene has been identified in MRSA and Enterococcus strains of human and animal origins [15-18]. Livestock is widely recognized as a reservoir of antimicrobial resistance genes [19].

In this study, we described for the first time the identification and characterization of poxtA gene in S. haemolyticus and S. saprophyticus isolates from pig and chicken farms in Guangdong province, China.

\section{Results}

\subsection{Identification of poxtA Gene in CoNS Isolates}

The poxt $A$ gene was detected in seven CoNS isolates in 2018 including five S. haemolyticus isolates (GDY8P33P, GDY8P50P, GDY8P58P, GDY8P60P and GDY8P80P all of pig origin) and two S. saprophyticus isolates (GDY8P136P of pig origin, GDH8C97P of feed origin) (Table 1).

Table 1. Background information on the 7 CoNS isolates carrying the poxtA gene.

\begin{tabular}{ccccc}
\hline Isolate & Sampling Time & Origin (Farm Type) & Species & MLST \\
\hline GDH8C97P & June 2018 & Feed sample (chicken farm A) & S. saprophyticus & - \\
GDY8P33P & December 2018 & swine nasal swab (pig farm D) & S. haemolyticus & ST64 \\
GDY8P50P & December 2018 & swine nasal swab (pig farm D) & S. haemolyticus & ST64 \\
GDY8058P & December 2018 & swine nasal swab (pig farm D) & S. haemolyticus & ST64 \\
GDY8P60P & December 2018 & swine nasal swab (pig farm D) & S. haemolyticus & ST64 \\
GDY8P80P & December 2018 & swine nasal swab (pig farm D) & S. haemolyticus & ST64 \\
GDY8P136P & December 2018 & swine nasal swab (pig farm D) & S. saprophyticus & - \\
\hline
\end{tabular}

MLST: “-" indicates that S. saprophyticus cannot be typed by MLST.

\subsection{Distribution of ARGs in poxtA-Positive CoNS Isolates and the Electrotransformants}

In total, 16 additional ARGs were detected among the poxt $A$-positive CoNS isolates (Figure 1). ARGs were widespread in the poxtA-positive isolates of both feed and pig origins. Except for the widely distributed resistance genes aadD, ant (6)-Ia, blaZ, mecA, lsa(E), $\ln u(\mathrm{~B}), \operatorname{erm}(\mathrm{C}), \operatorname{fexB}, \operatorname{tet}(\mathrm{L})$ and $d f r G$ in the poxt $A$-positive CoNS isolates, the distribution of individual ARG varied among the poxt A-positive CoNS isolates. For example, the $c f r$, tet $(\mathrm{M})$ and $\operatorname{aac}\left(6^{\prime}\right)-\operatorname{aph}\left(2^{\prime \prime}\right)$ genes were also identified in the poxtA-positive $S$. haemolyticus isolates (Figure 1). All poxtA-positive CoNS isolates were able to transfer the fexB, poxt $A$ and tet $(\mathrm{L})$ genes to $S$. aureus strain RN4220 (Table 2). In addition, the five poxt $A$-positive $S$. haemolyticus isolates were able to transfer the aadD gene to $S$. aureus strain RN4220 (Table 2).

\subsection{Antimicrobial Susceptibility}

Antimicrobial susceptibility testing showed that resistance rates of seven poxtApositive CoNS isolates to penicillin, cefoxitin, doxycycline, tetracycline, florfenicol, erythromycin and ciprofloxacin reached $100 \%$ (Table 2). Six (85.7\%) poxtA-positive CoNS isolates demonstrated resistance to enrofloxacin. All poxtA-positive CoNS isolates re- 
mained susceptible to linezolid, tedizolid, tigecycline, amikacin, gentamicin, rifampicin and vancomycin. All poxtA-positive CoNS isolates were able to transfer the florfenicol resistance to $S$. aureus strain RN4220. Two electrotransformants are erythromycin resistant (Table 2). In addition, the electrotransformants carrying $a a d D, \operatorname{poxt} A$ and $\operatorname{tet}(\mathrm{L})$ genes exhibited lower MICs of neomycin, kanamycin, linezolid, tedizolid, doxycycline and tetracycline compared with the donors (Table 2).

\subsection{Phylogenetic Relatedness of poxtA-Positive CoNS Isolates}

All poxtA-positive $S$. haemolyticus isolates derived from swine nasal swabs in pig farm D represented ST64 by MLST analysis and were closely related by phylogenetic analysis (Table 1 and Figure 1). In addition, 121 SNPs were identified in all poxtA-positive S. haemolyticus isolates (Figure 1). The S. saprophyticus isolates GDH8C97P recovered from feed in chicken farm A and GDY8P136P recovered from swine nasal swab in pig farm D shared 1957 SNPs difference (Figure 1).

\subsection{Plasmids Analysis}

S1-PFGE and WGS analysis confirmed that the poxt $A$ gene in the seven CoNS isolates and corresponding electrotransformants was located on plasmids ranging in size around $54.7 \mathrm{~kb}$ (Figure 2 and Figure S1). Plasmid pY80 carrying the poxt $A$ gene was $55,758 \mathrm{bp}$ in size and exhibited $<38 \%$ coverage with other plasmids in NCBI database, with an average GC content of $34.0 \%$. In total, 51 ORFs coding for proteins of $>50$ amino acids were identified (Figure 2). Except for the 14 ORFs encoding hypothetical proteins with no defined function, the products of the remaining 37 ORFs exhibited identities ranging from $76.3 \%$ to $100 \%$ to proteins with known functions, including antimicrobial resistance, heavy metal resistance, conjugative transfer or transposition, plasmid replication and other function (Figure 2).
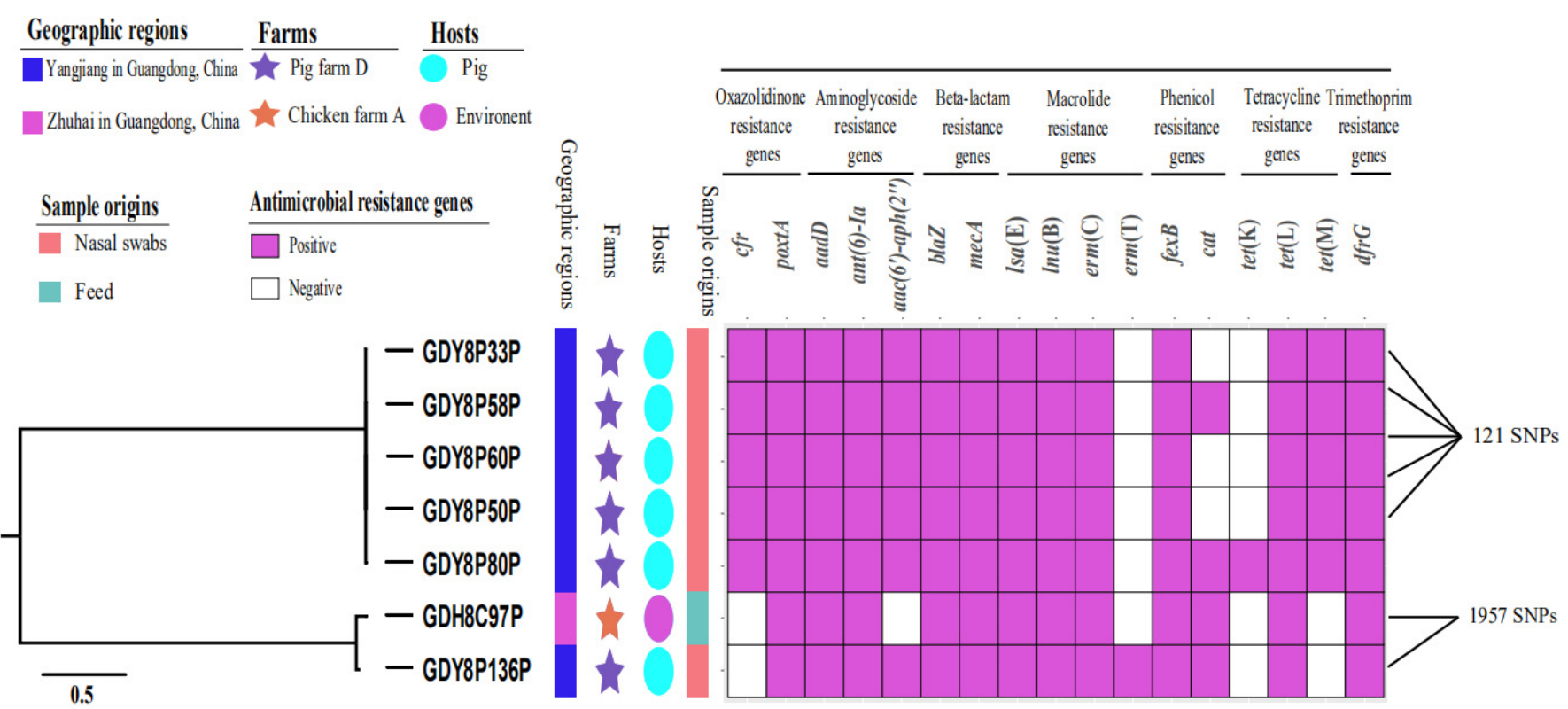

Figure 1. Genomic analysis of five Staphylococcus haemolyticus isolates carrying poxtA and cfr and two Staphylococcus saprophyticus isolates carrying poxt $A$ of various origins in Guangdong, China. Phylogenic tree was constructed using CSI Phylogeny 1.4. Sources of the isolates are indicated by different colors for geographic regions (squares), farms (stars), hosts (circles) and sample origins (squares). Antimicrobial resistance genes are indicated by the following method: purple, positive; white, negative. 
Table 2. Characterization of poxt $A$-positive strains, their electrotransformants and the recipient strain.

\begin{tabular}{|c|c|c|c|c|c|c|c|c|c|c|c|c|c|c|c|c|c|c|c|c|}
\hline \multirow{2}{*}{ Bacterial Isolate } & \multicolumn{19}{|c|}{ MICs (mg/L) } & \multirow{2}{*}{ Resistance Genes } \\
\hline & AMO & PEN & FOX & GEN & AMI & NEO & KAN & DOX & TET & TIG & FFC & ERY & RIF & VAN & CIP & ENR & LZD & TZD & SXT & \\
\hline S. aureus RN4220 & 0.125 & 0.125 & 2 & 0.25 & 1 & 0.25 & 0.25 & 0.125 & 0.5 & 0.06 & 2 & 0.25 & 0.008 & 1 & 0.5 & 0.25 & 0.5 & 0.06 & 0.25 & \\
\hline GDH8C97P & 8 & 16 & 8 & 0.125 & 0.25 & 1 & 0.25 & $>64$ & $>64$ & 0.25 & $>64$ & $>256$ & 0.015 & 1 & 4 & 16 & 0.5 & 0.06 & 0.5 & $\operatorname{aad} D, \operatorname{fex} B, \operatorname{poxt} A, \operatorname{tet}(\mathrm{L})$ \\
\hline GDY8P33P & 2 & 4 & 16 & 8 & 2 & 4 & 8 & $>64$ & $>64$ & 0.25 & $>64$ & $>256$ & 0.004 & 1 & $>64$ & 32 & 2 & 0.06 & 2 & $\operatorname{aad} D$, fexB, $\operatorname{poxt} A, \operatorname{tet}(\mathrm{L}), \operatorname{tet}(\mathrm{M})$ \\
\hline RN4220/pY33 & 0.06 & 0.125 & 2 & 0.125 & 2 & 1 & 0.25 & 1 & 2 & 0.125 & 32 & 0.125 & 0.008 & 1 & 0.5 & 0.125 & 0.5 & 0.06 & 0.25 & aadD, fexB, poxtA, tet $(\mathrm{L})$ \\
\hline GDY8P50P & 4 & 4 & 32 & 8 & 2 & 4 & 8 & 64 & $>64$ & 0.25 & $>64$ & $>256$ & 0.004 & 1 & $>64$ & $>64$ & 2 & 0.25 & 8 & $\operatorname{aad} D, \operatorname{fex} B, \operatorname{poxt} A$, tet $(\mathrm{L})$, tet $(\mathrm{M})$ \\
\hline RN4220/pY50 & 0.06 & 0.125 & 2 & 0.125 & 2 & 1 & 0.25 & 2 & 2 & 0.125 & 32 & 0.125 & 0.008 & 1 & 0.5 & 0.125 & 0.5 & 0.06 & 0.25 & aadD, fexB, poxt $A$, tet $(\mathrm{L})$ \\
\hline GDY8058P & 4 & 4 & 32 & 8 & 2 & 4 & 8 & 64 & $>64$ & 0.25 & $>64$ & $>256$ & 0.004 & 1 & $>64$ & $>64$ & 4 & 0.5 & 16 & $\operatorname{aad} D, \operatorname{fex} B, \operatorname{poxt} A$, tet $(\mathrm{L})$, tet $(\mathrm{M})$ \\
\hline GDY8P60P & 2 & 1 & 32 & 8 & 2 & 4 & 8 & 64 & 64 & 0.25 & $>64$ & $>256$ & 0.008 & 1 & $>64$ & $>64$ & 2 & 0.5 & 8 & aad $D$, fex $B, \operatorname{poxt} A$, tet $(\mathrm{L}), \operatorname{tet}(\mathrm{M})$ \\
\hline RN4220/pY60 & 0.06 & 0.125 & 2 & 0.125 & 2 & 1 & 0.25 & 1 & 1 & 0.25 & 32 & $>256$ & 0.002 & 1 & 0.5 & 0.125 & 0.5 & 0.06 & 0.25 & aadD, fexB, poxt $A$, tet $(\mathrm{L})$ \\
\hline GDY8P80P & 2 & 4 & 16 & 8 & 2 & 4 & 8 & 64 & $>64$ & 0.25 & $>64$ & $>256$ & 0.004 & 1 & $>64$ & $>64$ & 4 & 0.25 & 16 & $\operatorname{aadD}, \operatorname{fex} B, \operatorname{poxt} A, \operatorname{tet}(\mathrm{L}), \operatorname{tet}(\mathrm{M})$ \\
\hline RN4220/pY80 & 0.06 & 0.125 & 2 & 0.125 & 2 & 1 & 0.25 & 1 & 2 & 0.25 & 32 & 0.125 & 0.008 & 1 & 0.5 & 0.125 & 0.5 & 0.06 & 0.25 & aadD, fexB, poxt $A$, tet $(\mathrm{L})$ \\
\hline GDY8P136P & 16 & 16 & 16 & 8 & 0.5 & 4 & 8 & 32 & 64 & 0.25 & 64 & $>256$ & 0.03 & 2 & 4 & 2 & 2 & 0.25 & 1 & $\operatorname{aad} D, \operatorname{fex} B, \operatorname{poxt} A, \operatorname{tet}(\mathrm{L})$ \\
\hline RN4220/pY136 & 0.06 & 0.125 & 1 & 0.125 & 0.5 & 0.25 & 0.25 & 0.06 & 2 & 0.03 & 16 & 0.125 & 0.002 & 1 & 0.25 & 0.125 & 0.5 & 0.06 & 0.25 & fexB, poxt $A$, tet $(\mathrm{L})$ \\
\hline
\end{tabular}

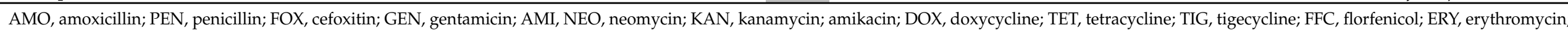

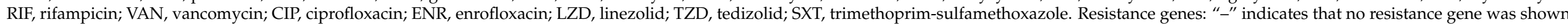

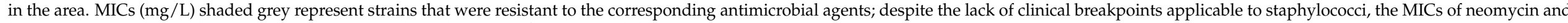
kanamycin were detected in poxtA-positive strains, their electrotransformants and the recipient strain S. aureus RN4220. 


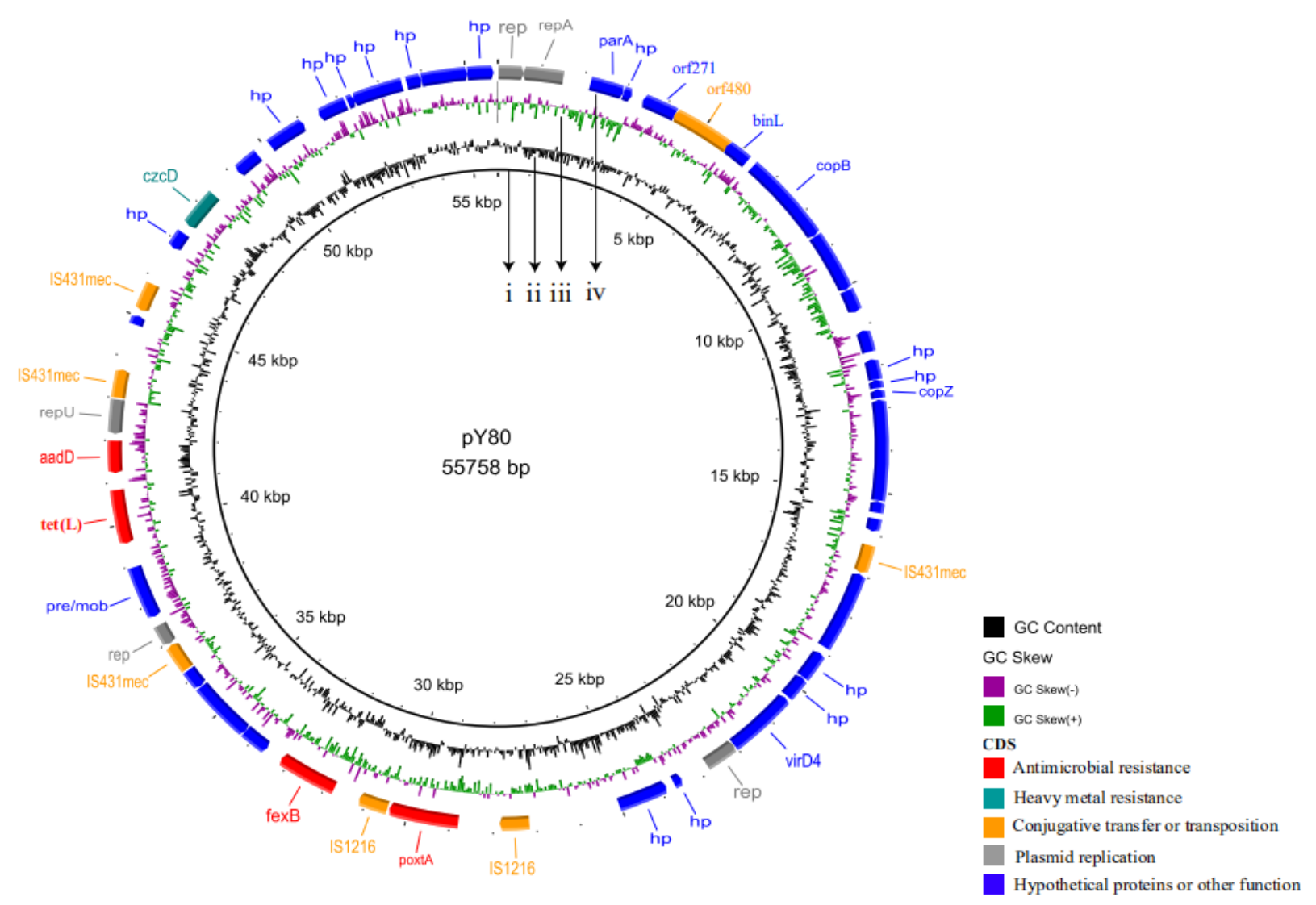

Figure 2. Annotation of plasmid pY80. Circles were displayed (inside to outside) (i) size in bp; (ii) GC content; (iii) GC skew; (iv) positions and directions of predicted coding sequences are indicated by colored arrows according to their predicted functions. Red arrows represent resistance genes, teal arrows represent heavy meatal resistance genes, orange arrows represent genes involved in transfer or transposition, gray arrows represent plasmid replication genes, blue arrows represent genes of unknown functions or other functions.

\subsection{Genetic Environment of poxtA Gene}

The poxtA-carrying segments (IS1216-poxtA-IS1216-fexB-IS431mec-tet(L)-aadD-IS431mec) of $17287 \mathrm{bp}$ in plasmid pY80 of pig origin) were selected to conduct comparative analysis with other poxtA-carrying segments. The IS1216-poxtA-IS1216 segment of $4130 \mathrm{bp}$ showed $>98 \%$ identity to corresponding sequences in two Enterococcus hirae plasmids (pHDC142.27K and pfas4-1 both of pig origins), two Enterococcus faecalis plasmids (pM18/0011 of human origin and pC10 of pig origin), 10 Enterococcus faecium plasmids (pSDGJQ5 of chicken origin, pM160954 of human origin, pE1077-23 of pig origin, pSCBC1 of pig origin, pSDGJP3 of pig origin, pYN2-1 of pig origin, pHN11 of pig origin, pGZ8 of pig origin, pSC3-1 of chicken origin and pC25-1 of pig origin) and the genome of S. aureus AOUC-0915 of human origin (Figure 3). In addition, the IS1216-poxtA segment of $2363 \mathrm{bp}$ showed $>98 \%$ identity to corresponding sequences in the genomes of Enterococcus faecium P36 of pig origin and Pediococcus acidilactici BCC1 of chicken origin (Figure 3). The $11951 \mathrm{bp}$ region (fexB-IS431mec-tet(L)-aadD-IS431mec) downstream of IS1216-poxtA-IS1216 in plasmid pY80 was identified. Within this region, the $4200 \mathrm{bp}$ segment (tet(L)-aadD-IS431mec) showed $>98 \%$ identity to the $S$. aureus plasmid (pERGB of human origin) (Figure 3). The poxtAcarrying fragments that often harbor additional resistance genes such as $f e x B, \operatorname{tet}(\mathrm{L})$ and tet(M) were identified in different bacterial species (Figure 3). 


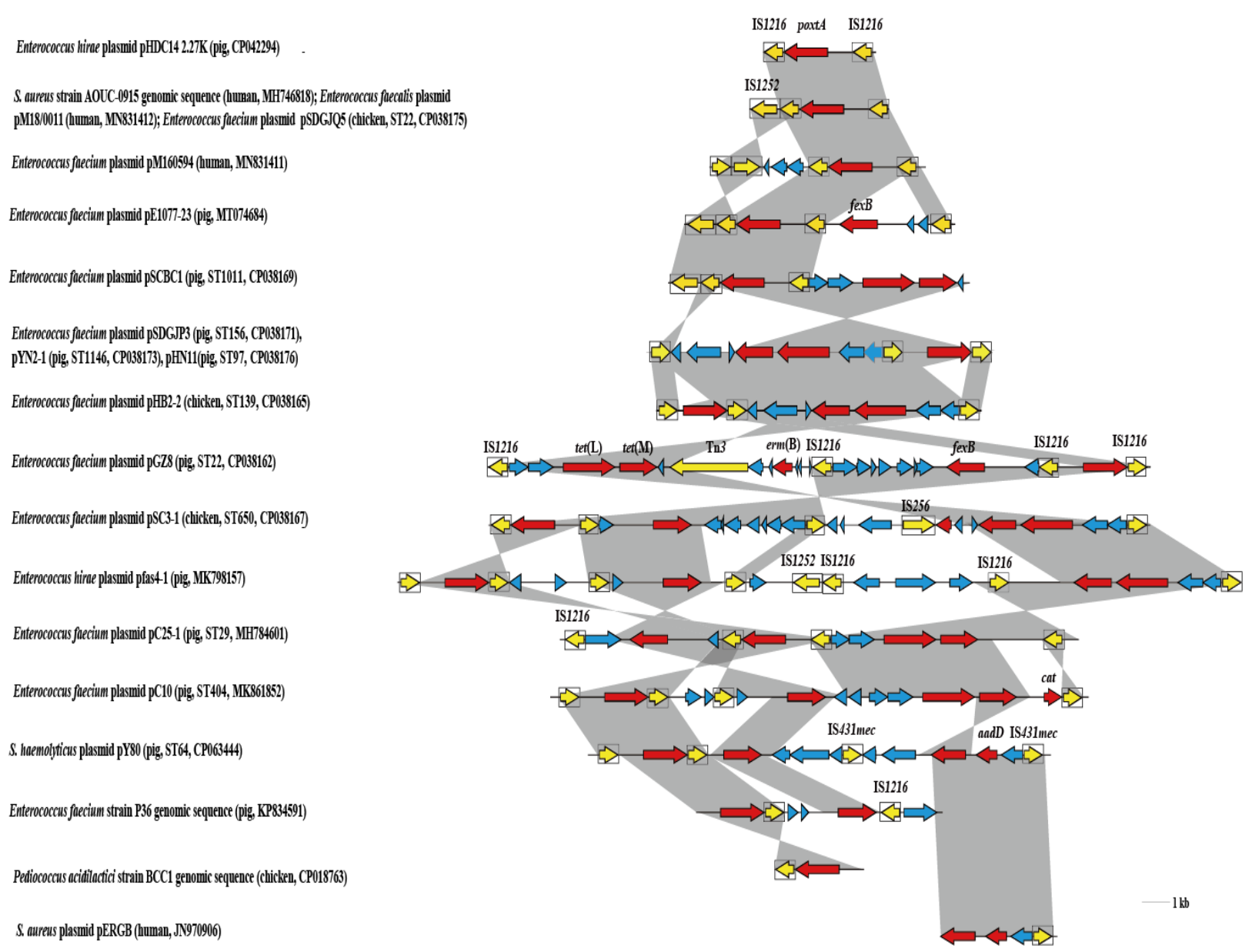

Figure 3. Comparison of the genetic contexts of poxtA in plasmid pY80 investigated in this study with corresponding sequences in other plasmids and genomic DNA. Arrows indicate the positions and orientations of the genes. Antimicrobial resistance genes are shown in red. Mobile element regions are underlined in yellow. Insertion sequences are indicated as boxes, with the arrow inside the box showing the transposase gene. Genes with unknown functions and other functions are shown in light blue. Regions of $>98 \%$ nucleotide sequence identity are shaded grey. $\Delta$ indicates an incomplete gene.

\section{Discussion}

CoNS are recognized as significant opportunistic pathogens that cause infections in humans and animals $[4,5]$, and CoNS carrying important antimicrobial resistance genes such as oxazolidinone resistance genes could pose a huge burden on the healthcare system and breeding industry [10]. The transferable oxazolidinone resistance gene poxt $A$ in different enterococci was the most recently reported [17]. Attention should be paid to the fact that the poxtA gene was originally detected in a linezolid-resistant MRSA strain [16]. Therefore, there was a risk that the poxtA could spread to other bacterial strains. The observation that the poxtA gene was mainly detected in S. haemolyticus and S. saprophyticus isolates might suggest an S. haemolyticus and S. saprophyticus reservoir. In this study, the results indicated that poxtA-positive ST64-S. haemolyticus isolates from swine nasal swabs in a pig farm shared low SNPs difference and were closely related, and that poxtA-positive S. saprophyticus isolates from a pig farm and a chicken farm shared a high SNPs difference. Therefore, ST64-S. haemolyticus isolates carrying poxtA can spread among pigs in the pig farm, and the potential spread of $S$. saprophyticus isolates carrying poxt $A$ between the pig farm and chicken farm should arouse people's attention.

It was reported that IS1216 played a major role in the processes of aiding the dissemination and persistence of poxt $A$ among enterococci [20]. The presence of these homologous gene regions (IS1216-poxtA-IS1216 or IS1216-poxtA) in Staphylococcus spp., Enterococcus spp. and Pediococcus spp. confirmed that IS1216 was closely related to the spread of poxtA 
among these Gram-positive bacteria with different genetic and source backgrounds. This was a further reminder that the poxt $A$ gene might have spread widely in these bacteria. It has been found from reported studies that most of these homologous gene regions are located on transferable plasmids which often harbor additional resistance genes such as the tetracycline resistance genes tet $(\mathrm{M})$ and tet $(\mathrm{L})$, and the phenicol exporter gene fexB [15-18]. In this study, plasmid pY80 carried heavy metal resistance gene $c z c D$ and aminoglycosidemodifying enzyme gene $a a d D$ in addition to tet $(\mathrm{L}), f e x B$ and $\operatorname{poxt} A$ genes. The co-occurrence of poxt $A$ with other antimicrobial and heavy metal resistance genes on the transferable plasmids may lead to the co-selection of $\operatorname{poxt} A$, contributing to its persistence and accelerating its dissemination [15]. The poxt $A$ gene was identified in the new plasmid. Once the poxt $A$ gene is inserted into a plasmid with strong transmission ability, it will bring great difficulties to control the further transmission of the poxt $A$ gene. In China, florfenicol has been widely used in food-producing animals [21]. It was reported that the emergence of oxazolidinone resistance genes such as poxt $A$ is closely related to the use of florfenicol in breeding farms [22]. Antimicrobial susceptibility testing showed that all donors and electrotransformants were resistant to florfenicol, indicating poxt $A$-positive plasmids could be directly selected by florfenicol. The phenomenon that the strains and electrotransformants investigated in this study were phenotypically oxazolidinone-susceptible despite the fact that they carry up to two oxazolidinone resistance genes ( $c f r$ and poxt $A$ ) is very interesting. This may be due to the possibility that $c f r$ was not transcribed [23] and poxt $A$ played a relatively low role on oxazolidinone susceptibility [16]. The fact that electrotransformants with $a a d D$ and tet(L) genes did not show resistance to kanamycin, neomycin, doxycycline and tetracycline might be related to the silencing of these genes. It is easy for people to ignore the resistance genes without corresponding drug-resistant phenotypes, resulting in the widespread spread of them [24]. That antimicrobial agents used in livestock could exert selective pressures on bacteria [25] and all the poxt $A$-positive CoNS isolates exhibited multidrug resistance and carried additional resistance genes should account for the spread of poxt $A$ gene in the CoNS isolates [15].

\section{Materials and Methods}

\subsection{Bacterial Isolations and Detection of poxtA Gene}

A total of 778 CoNS isolates were collected from 1 chicken farm, 15 pig farms and 18 duck farms in Guangdong, China, between 2018-2020. Isolates were recovered from $34.6 \%(9 / 26)$ of human nasal swabs, 58.9\% (353/599) of swine nasal swabs, 39.8\% (33/83) of feed samples, $47.5 \%$ (94/198) of pond water samples, 34.3\% (35/102) of soil samples, $65.4 \%(51 / 78)$ of airborne dust samples and animal 52.3\% (203/388) of viscera samples.

All isolates were screened for the presence of poxt $A$ by PCR using previously described primers [15]. Species identification was performed using MALDI-TOF MS (Bruker Daltonik $\mathrm{GmbH}$, Bremen, Germany) and further confirmed by $16 \mathrm{~S}$ rDNA sequence analysis.

\subsection{Molecular Epidemiology Analysis and Transformation Assays}

Multilocus sequence typing (MLST) was conducted for identification of clonal correlation of the poxtA-positive S. haemolyticus (http:/ / www.shaemolyticus.mlst.net Accessed on: 29 January 2021) [26]. Plasmid DNA from all poxtA-positive CoNS isolates was extracted using a Qiagen Prep Plasmid Midi Kit (Qiagen, Hilden, Germany) and transferred into a recipient $S$. aureus strain RN4220 by electroporation using Gene Pulser apparatus (Bio-Rad, Hercules, CA, United States) [27]. Electrotransformants were selected on brain heart infusion (BHI) agar containing $10 \mu \mathrm{g} / \mathrm{mL}$ of florfenicol. Electrotransformants were further confirmed for the presence of poxt $A$ gene by PCR analysis. The successful electrotransformants were further screened for the presence of $a a d D, f e x B$, tet $(\mathrm{L})$ and tet $(\mathrm{M})$ genes by PCR. 


\subsection{Antimicrobial Susceptibility Testing}

All poxtA-positive CoNS isolates and corresponding electrotransformants were investigated for their MICs of florfenicol, linezolid, tedizolid, amoxicillin, penicillin, cefoxitin, doxycycline, tetracycline, tigecycline, gentamicin, amikacin, neomycin, kanamycin, erythromycin, ciprofloxacin, enrofloxacin, rifampicin, vancomycin and trimethoprimsulfamethoxazole by broth microdilution following the recommendations given in CLSI documents VET01-S2 and M100-S30 [28,29]. Staphylococcus aureus ATCC 29213 was used as the quality control strain.

\subsection{S1-PFGE and WGS Analysis}

Genomic DNA of all poxtA-positive CoNS isolates and corresponding electrotransformants were digested with S1 endonuclease and separated by PFGE as previously described [30]. Whole-cell DNA of all poxtA-positive CoNS isolates were prepared using the HiPure Bacterial DNA Kit (Magen, Guangzhou, China), following the manufacturer's instructions, and then preceded by library construction on Novaseq 6,000 sequencing platform, which produced 150 bp paired-end reads (Novogene Company, Beijing, China). Novaseq sequences were assembled using CLC Genomics Workbench 10 (CLC Bio, Aarhus, Denmark). The GDY8P80P isolate carrying poxt $A$ and $c f r$ genes was further used for whole-genome sequencing on PacBio RS II sequencing platform (Biochip Company, Tianjin, China). Pacbio sequences were assembled using hierarchical genome-assembly process [31]. The assembled Pacbio sequences were corrected through Burrows-Wheeler Aligner's Smith-Waterman Alignment (BWA-SW) software to ensure their integrity according to Novaseq sequences [32]. The plasmids carrying poxt $A$ were annotated using the Rapid Annotation of microbial genomes using Subsystems Technology annotation server (http:/ / rast.nmpdr.org/ Accessed on: 29 January 2021) [33]. Acquired resistance genes (ARGs) were identified in the genomes using ResFinder 4.0 [34]. The genetic comparison of the poxtA gene from different species was generated using Easyfig 2.1 [35]. Based on the draft genome sequences, a phylogenic tree was constructed for all sequenced poxt $A$-positive CoNS isolates by CSI Phylogeny 1.4 (https:// cge.cbs.dtu.dk/services/CSIPhylogeny/ Accessed on: 29 January 2021), with the genome of GDY8P50P used as a reference. The tree was visualized using software Fig Tree 1.4.2. Single nucleotide polymorphism (SNP) divergence among various isolates carrying poxt $A$ was calculated using snippy (https:// www.github.com/heilaaks/snippy/Accessed on: 29 January 2021).

\subsection{Nucleotide Sequence Accession Numbers}

The complete sequences of strains GDH8C97P, GDY8P33P, GDY8P50P, GDY8P58P, GDY8P60, GDY8P80P and GDY8P136P have been deposited in GenBank under accession numbers JADICE000000000, JADQVZ000000000, JADICF000000000, JADICG000000000, JADICH000000000, JADICI000000000 and JADICJ000000000, respectively. The complete sequence of plasmid pY 80 have been deposited in GenBank under accession numbers CP063444.

\subsection{Ethical Considerations}

The study was approved by the South China Agriculture University (SCAU) Animal Ethics Committee. The research was conducted in strict accordance with Section 20 of the Animal Diseases Act of 1984 (Act No 35 of 1984) and the Declaration of Helsinki, and was approved by the SCAU Institutional Animal Care and Use Committee.

\section{Conclusions}

In conclusion, this is the first study to report on the presence of the poxt $A$ gene in livestock-derived S. haemolyticus and S. saprophyticus. The presence of IS1216-poxtA-IS1216 in Staphylococcus spp., Enterococcus spp. and Pediococcus spp. with different genetic and source backgrounds indicated an important role of IS1216 in the dissemination of poxt $A$. Moreover, the co-occurrence of poxt $A$ with other antimicrobial and heavy metal resistance 
genes on the transferable plasmids may lead to the co-selection of $p o x t A$, contributing to its persistence and accelerating its dissemination even in the absence of direct selective pressure by the use of phenicols, tetracyclines and oxazolidinones. Attention should be paid to the potential risks of the transfer of the plasmid-borne poxt $A$ from enterococci and staphylococci to other Gram-positive bacteria. Therefore, routine surveillance for the spread of poxt $A$ in different Gram-positive bacteria and the prudent use of antimicrobial agents in food-producing animals are urgently warranted.

Supplementary Materials: The following are available online at https:/ /www.mdpi.com/article/10 .3390 / pathogens10050601/s1, Figure S1: Location of the poxtA-carrying plasmids in seven CoNS isolates and corresponding electrotransformants by S1-PFGE. Lanes M contain the XbaI pattern of Salmonella braenderup $\mathrm{H} 9812$ with the fragment sizes given in kilobases on the left-hand and righthand sides; lanes 1 to 14 represent GDH8C97P, RN4220/pH97, GDY8P33P, RN4220/pY33, GDY8P50P, RN4220/pY50, GDY8P58P, RN4220/pY58, GDY8P60P, RN4220/pY60, GDY8P80P, RN4220/pY80, GDY8P136P and RN4220/pY136, respectively.

Author Contributions: Conceptualization, L.C., J.-X.H., C.L., J.L., Z.-B.M., Z.-Y.T., Y.-F.L. and Z.-L.Z.; methodology, L.C., J.-X.H., Z.-B.M. and Z.-Y.T.; software, L.C. and Z.-B.M.; validation, Y.-F.L. and Z.-L.Z.; formal analysis, L.C.; investigation, L.C., J.-X.H., Z.-B.M. and Z.-Y.T.; data curation, L.C. and Z.-B.M.; writing-original draft preparation, L.C.; writing-review and editing, L.C., J.-X.H., C.L., J.L., Z.-B.M., Z.-Y.T., Y.-F.L. and Z.-L.Z.; supervision and project administration, Y.-F.L. and Z.-L.Z.; funding acquisition, Z.-L.Z. All authors have read and agreed to the published version of the manuscript.

Funding: This work was financially supported by the National Key R \& D Program of China (No. 2018YFD0500300).

Institutional Review Board Statement: The study was conducted according to the guidelines of the Declaration of Helsinki, and approved by the South China Agricultural University (SCAU) Animal Ethics Committee. The field sampling protocols, samples collected from livestock, strain isolation, and the research were conducted in strict accordance with Section 20 of the Animal Diseases Act of 1984 (Act No 35 of 1984), and were approved with the SCAU Institutional Animal Care and Use Committee.

Informed Consent Statement: Informed consent was obtained from all subjects involved in the study.

Data Availability Statement: Not applicable.

Conflicts of Interest: The authors declare no conflict of interest.

\section{References}

1. Al Tayyar, I.A.; Al-Zoubi, M.S.; Hussein, E.; Khudairat, S.; Sarosiekf, K. Prevalence and antimicrobial susceptibility pattern of coagulase-negative staphylococci (CoNS) isolated from clinical specimens in Northern of Jordan. Iran. J. Microbiol. 2015, 7, 294-301.

2. Czekaj, T.; Ciszewski, M.; Szewczyk, E.M. Staphylococcus haemolyticus-An emerging threat in the twilight of the antibiotics age. Microbiology 2015, 161, 2061-2068. [CrossRef]

3. Argemi, X.; Hansmann, Y.; Prola, K.; Prevost, G. Coagulase-Negative Staphylococci Pathogenomics. Int. J. Mol. Sci. 2019, 20, 1215. [CrossRef]

4. Venugopal, N.; Mitra, S.; Tewari, R.; Ganaie, F.; Shome, R.; Rahman, H.; Shome, B.R. Molecular detection and typing of methicillinresistant Staphylococcus aureus and methicillin-resistant coagulase-negative staphylococci isolated from cattle, animal handlers, and their environment from Karnataka, Southern Province of India. Vet. World. 2019, 12, 1760-1768. [CrossRef] [PubMed]

5. Teeraputon, S.; Santanirand, P.; Wongchai, T.; Songjang, W.; Lapsomthob, N.; Jaikrasun, D.; Toonkaew, S.; Tophon, P. Prevalence of methicillin resistance and macrolide-lincosamide-streptogramin B resistance in Staphylococcus haemolyticus among clinical strains at a tertiary-care hospital in Thailand. New Microbes New Infect. 2017, 19, 28-33. [CrossRef]

6. Farrell, D.J.; Mendes, R.E.; Bensaci, M. In vitro activity of tedizolid against clinical isolates of Staphylococcus lugdunensis and Staphylococcus haemolyticus from Europe and the United States. Diagn. Microbiol. Infect. Dis. 2019, 93, 85-88. [CrossRef] [PubMed]

7. Paiva-Santos, W.; Sousa, V.S.; Giambiagi-deMarval, M. Occurrence of virulence-associated genes among Staphylococcus saprophyticus isolated from different sources. Microb. Pathog. 2018, 119, 9-11. [CrossRef] [PubMed]

8. Douros, A.; Grabowski, K.; Stahlmann, R. Drug-drug interactions and safety of linezolid, tedizolid, and other oxazolidinones. Expert Opin. Drug Metab. Toxicol. 2015, 11, 1849-1859. [CrossRef] [PubMed] 
9. $\quad$ Bender, J.K.; Cattoir, V.; Hegstad, K.; Sadowy, E.; Coque, T.M.; Westh, H.; Hammerum, A.M.; Schaffer, K.; Burns, K.; Murchan, S.; et al. Update on prevalence and mechanisms of resistance to linezolid, tigecycline and daptomycin in enterococci in Europe: Towards a common nomenclature. Drug Resist. Updates 2018, 40, 25-39. [CrossRef]

10. Tewhey, R.; Gu, B.; Kelesidis, T.; Charlton, C.; Bobenchik, A.; Hindler, J.; Schork, N.J.; Humphries, R.M. Mechanisms of Linezolid Resistance among Coagulase-Negative Staphylococci Determined by Whole-Genome Sequencing. mBio 2014, 5, e00894-14. [CrossRef]

11. Deshpande, L.M.; Castanheira, M.; Flamm, R.K.; Mendes, R.E. Evolving oxazolidinone resistance mechanisms in a worldwide collection of enterococcal clinical isolates: Results from the SENTRY Antimicrobial Surveillance Program. J. Antimicrob. Chemother. 2018, 73, 2314-2322. [CrossRef] [PubMed]

12. Hao, H.H.; Sander, P.; Iqbal, Z.; Wang, Y.L.; Cheng, G.Y.; Yuan, Z.H. The Risk of Some Veterinary Antimicrobial Agents on Public Health Associated with Antimicrobial Resistance and their Molecular Basis. Front. Microbiol. 2016, 7, 1626. [CrossRef]

13. Shen, J.Z.; Wang, Y.; Schwarz, S. Presence and dissemination of the multiresistance gene cfr in Gram-positive and Gram-negative bacteria. J. Antimicrob. Chemother. 2013, 68, 1697-1706. [CrossRef]

14. Huang, J.H.; Chen, L.; Wu, Z.W.; Wang, L.P. Retrospective analysis of genome sequences revealed the wide dissemination of optrA in Gram-positive bacteria. J. Antimicrob. Chemother. 2017, 72, 614-616. [CrossRef]

15. Huang, J.H.; Wang, M.L.; Gao, Y.; Chen, L.; Wang, L.P. Emergence of plasmid-mediated oxazolidinone resistance gene poxtA from CC17 Enterococcus faecium of pig origin. J. Antimicrob. Chemother. 2020, 75, 1359-1361. [CrossRef] [PubMed]

16. Antonelli, A.; D'Andrea, M.M.; Brenciani, A.; Galeotti, C.L.; Morroni, G.; Pollini, S.; Varaldo, P.E.; Rossolini, G.M. Characterization of $\operatorname{poxt} A$, a novel phenicol-oxazolidinone-tetracycline resistance gene from an MRSA of clinical origin. J. Antimicrob. Chemother. 2018, 73, 1763-1769. [CrossRef]

17. Egan, S.A.; Shore, A.C.; O'Connell, B.; Brennan, G.I.; Coleman, D.C. Linezolid resistance in Enterococcus faecium and Enterococcus faecalis from hospitalized patients in Ireland: High prevalence of the MDR genes optrA and poxt $A$ in isolates with diverse genetic backgrounds. J. Antimicrob. Chemother. 2020, 75, 1704-1711. [CrossRef]

18. Freitas, A.R.; Tedim, A.P.; Duarte, B.; Elghaieb, H.; Abbassi, M.S.; Hassen, A.; Read, A.; Alves, V.; Novais, C.; Peixe, L. Linezolidresistant (Tn6246::fexB-poxtA) Enterococcus faecium strains colonizing humans and bovines on different continents: Similarity without epidemiological link. J. Antimicrob. Chemother. 2020, 75, 2416-2423. [CrossRef] [PubMed]

19. Xiong, W.G.; Wang, Y.L.; Sun, Y.X.; Ma, L.P.; Zeng, Q.L.; Jiang, X.T.; Li, A.T.; Zeng, Z.L.; Zhang, T. Antibiotic-mediated changes in the fecal microbiome of broiler chickens define the incidence of antibiotic resistance genes. Microbiome 2018, 6, 34. [CrossRef] [PubMed]

20. Fioriti, S.; Morroni, G.; Coccitto, S.N.; Brenciani, A.; Antonelli, A.; Di Pilato, V.; Baccani, I.; Pollini, S.; Cucco, L.; Morelli, A.; et al. Detection of oxazolidinone resistance genes and characterization of genetic environments in enterococci of swine origin, Italy. Microorganisms 2020, 8, 2021. [CrossRef] [PubMed]

21. Wang, Y.; Lv, Y.; Cai, J.C.; Schwarz, S.; Cui, L.Q.; Hu, Z.D.; Zhang, R.; Li, J.; Zhao, Q.; He, T.; et al. A novel gene, optrA, that confers transferable resistance to oxazolidinones and phenicols and its presence in Enterococcus faecalis and Enterococcus faecium of human and animal origin. J. Antimicrob. Chemother. 2015, 70, 2182-2190. [CrossRef]

22. Wang, Y.Y.; Li, X.W.; Fu, Y.L.; Chen, Y.Q.; Wang, Y.; Ye, D.Y.; Wang, C.F.; Hu, X.; Zhou, L.; Du, J.J.; et al. Association of florfenicol residues with the abundance of oxazolidinone resistance genes in livestock manures. J. Hazard. Mater. 2020, $399,123059$. [CrossRef]

23. Brenciani, A.; Morroni, G.; Vincenzi, C.; Manso, E.; Mingoia, M.; Giovanetti, E.; Varaldo, P.E. Detection in Italy of two clinical Enterococcus faecium isolates carrying both the oxazolidinone and phenicol resistance gene optrA and a silent multiresistance gene cfr. J. Antimicrob. Chemother. 2016, 71, 1118-1119. [CrossRef] [PubMed]

24. Liu, Y.; Wang, Y.; Schwarz, S.; Wang, S.L.; Chen, L.R.; Wu, C.M.; Shen, J.Z. Investigation of a multiresistance gene $c f r$ that fails to mediate resistance to phenicols and oxazolidinones in Enterococcus faecalis. J. Antimicrob. Chemother. 2014, 69, 892-898. [CrossRef]

25. Subbiah, M.; Mitchell, S.M.; Ullman, J.L.; Call, D.R. $\beta$-Lactams and florfenicol antibiotics remain bioactive in soils while ciprofloxacin, neomycin, and tetracycline are neutralized. Appl. Environ. Microbiol. 2011, 77, 7255-7260. [CrossRef]

26. Tenover, F.C.; Arbeit, R.D.; Goering, R.V.; Mickelsen, P.A.; Murray, B.E.; Persing, D.H.; Swaminathan, B. Interpreting chromosomal DNA restriction patterns produced by pulsed-field gel electrophoresis: Criteria for bacterial strain typing. J. Clin. Microbiol. 1995, 33, 2233-2239. [CrossRef]

27. Li, S.M.; Zhou, Y.F.; Li, L.; Fang, L.X.; Duan, J.H.; Liu, F.R.; Liang, H.Q.; Wu, Y.T.; Gu, W.Q.; Liao, X.P.; et al. Characterization of the Multi-Drug Resistance Gene cfr in Methicillin-Resistant Staphylococcus aureus (MRSA) Strains Isolated from Animals and Humans in China. Front. Microbiol. 2018, 9, 2925. [CrossRef]

28. Clinical and Laboratory Standards Institute. Performance Standards for Antimicrobial Disk and Diffusion Susceptibility Tests for Bacteria Isolated from Animals: Second Informational Supplement VET01-S2; CLSI: Wayne, PA, USA, 2013.

29. Clinical and Laboratory Standards Institute. Performance Standards for Antimicrobial Susceptibility Testing: Twenty-Sixth Informational Supplement M100-S30; CLSI: Wayne, PA, USA, 2020.

30. Barton, B.M.; Harding, G.P.; Zuccarelli, A.J. A general method for detecting and sizing large plasmids. Anal. Biochem. 1995, 226, 235-240. [CrossRef] [PubMed] 
31. Chin, C.S.; Alexander, D.H.; Marks, P.; Klammer, A.A.; Drake, J.; Heiner, C.; Clum, A.; Copeland, A.; Huddleston, J.; Eichler, E.E.; et al. Nonhybrid, finished microbial genome assemblies from long-read SMRT seqfuencing data. Nat. Methods 2013, 10, 563-569. [CrossRef] [PubMed]

32. Li, H.; Durbin, R. Fast and accurate long-read alignment with Burrows-Wheeler transform. Bioinformatics 2010, 26, 589-595. [CrossRef]

33. Overbeek, R.; Olson, R.; Pusch, G.D.; Olsen, G.J.; Davis, J.J.; Disz, T.; Edwards, R.A.; Gerdes, S.; Parrello, B.; Shukla, M.; et al. The SEED and the Rapid Annotation of Microbial Genomes Using Subsystems Technology (RAST). Nucleic Acids Res. 2014, 42, D206-D214. [CrossRef] [PubMed]

34. Zankari, E.; Hasman, H.; Cosentino, S.; Vestergaard, M.; Rasmussen, S.; Lund, O.; Aarestrup, F.M.; Larsen, M.V. Identification of acquired antimicrobial resistance genes. J. Antimicrob. Chemother. 2012, 67, 2640-2644. [CrossRef]

35. Sullivan, M.J.; Petty, N.K.; Beatson, S.A. Easyfig: A genome comparison visualizer. Bioinformatics 2011, 27, 1009-1010. [CrossRef] [PubMed] 